\title{
Barreras para la Movilización Temprana en una Unidad de Cuidados Intensivos Pediátricos en México
}

\author{
Robert Alexander Jones Baro, PT \\ Jhonatan Surihém Pérez Duarte, PT
}

Licenciatura en Fisioterapia, Universidad Autónoma de Querétaro, México

Miguel Ángel Martínez Camacho, PT

Licenciatura en Fisioterapia, Universidad Autónoma de Querétaro, México

Maestría en Ciencias de la Rehabilitación en el Movimiento Humano,

Universidad Autónoma de Querétaro, México

Grupo AVENTHO Ventilación Mecánica

Ivonne Villanueva Díaz, MD

Unidad de Terapia Intensiva del Hospital de Especialidades del Niño y la Mujer-SESEQ, Querétaro, México, Unidad de Terapia Intensiva del Hospital Infantil Teletón de Oncología, Querétaro, México

Sandra Mariana Chávez Monjarás, PT

Licenciatura en Fisioterapia, Universidad Autónoma de Querétaro, México

Maestría en Ciencias de la Rehabilitación en el Movimiento Humano,

Universidad Autónoma de Querétaro, México

\section{Resumen}

La movilización temprana (MT) se define como la implementación de actividad física en pacientes críticamente enfermos entre el segundo y quinto día de ingreso en la Unidad de Cuidados Intensivos (UCI). La MT se ha descrito como una práctica segura, factible y con grandes beneficios a corto y largo plazo. Para implementar un protocolo de MT el equipo multidisciplinario debe identificar y superar las barreras de percepción para la práctica de la misma. Algunas de las barreras más mencionadas son la falta de equipamiento, la pobre o nula cantidad de guías de práctica, la sedación pediátrica y las ordenes médicas. Objetivo: Describir las barreras dentro de la Unidad de Cuidados Intensivos Pediátricos (UCIP) Diseño: Estudio descriptivo transversal. Método: Se aplicó un instrumento para identificar las principales barreras a la MT a 28 profesionales que laboraran en la UCIP del Hospital de Especialidades del Niño y la Mujer en Querétaro (HENM), México. Resultados: La única barrera importante observada para la 
movilización de los pacientes en la UCIP es la ausencia de equipo y mobiliario. Otras barreras pueden ser observadas, más no descritas, como la carencia de personal fisioterapéutico dentro de la UCIP. Conclusión: La MT es aceptada como una intervención importante dentro de la UCIP. Si queremos describir completamente las barreras con respecto a la misma, las UCIP deberán ser equipadas de forma adecuada. Serán necesarios más estudios complementarios para darle seguimiento a la actividad física dentro de las terapias intensivas.

Palabras Clave: Movilización Temprana, Unidad de Cuidados Intensivos Pediátricos, Barreras en la Movilización

\title{
Barriers for Early Mobilization in a Pediatric Intensive Care Unit in Mexico
}

\author{
Robert Alexander Jones Baro, PT \\ Jhonatan Surihém Pérez Duarte, PT
}

Licenciatura en Fisioterapia, Universidad Autónoma de Querétaro, México

Miguel Ángel Martínez Camacho, PT

Licenciatura en Fisioterapia, Universidad Autónoma de Querétaro, México

Maestría en Ciencias de la Rehabilitación en el Movimiento Humano,

Universidad Autónoma de Querétaro, México

Grupo AVENTHO Ventilación Mecánica

Ivonne Villanueva Díaz, MD

Unidad de Terapia Intensiva del Hospital de Especialidades del Niño y la Mujer-SESEQ, Querétaro, México, Unidad de Terapia Intensiva del Hospital Infantil Teletón de Oncología, Querétaro, México

Sandra Mariana Chávez Monjarás, PT

Licenciatura en Fisioterapia, Universidad Autónoma de Querétaro, México

Maestría en Ciencias de la Rehabilitación en el Movimiento Humano,

Universidad Autónoma de Querétaro, México

Abstract

Early mobilization (EM) is defined as the application of physical activity within the first two to five days of critical illness or injury in an Intensive Care Unit (ICU). EM is proven to be a safe practice; it is also been described as feasible and with great short to long term benefits. In order to 
implement EM in the ICU the multidisciplinary team must identify and overcome perception barriers. Some of the most mentioned barriers in the pediatric ICU are the lack of equipment, the poor or none practice guidelines, pediatric sedation protocols and physician orders. Objective: Describe the barriers limiting the EM practice in a pediatric intensive care unit (PICU). Design: A transversal descriptive study. Method: A total of 28 professional working in the PICU of the Hospital of Children and Women Specialties in Querétaro, México answered a survey to identify the most important barriers for EM. Results: The only important barrier observed was the lack of equipment perceived by the multidisciplinary team. Other barriers where noticed but not motioned such as the low numbers of physical therapists working in the PICU. Conclusion: EM is accepted as an important part of the treatments involved in the PICU. If we want to completely describe Mexican barriers towards the implementation of EM protocols the ICU must be better equipped. Further studies will be needed in order to have a follow up on the physical activity implemented in Mexican PICUs.

Keywords: Early mobilization, Pediatric Intensive Care Unit, Barriers for mobilization

\section{Introduccion}

La movilización temprana (MT) se ha definido como la implementación de actividad física en pacientes críticamente enfermos dentro del día 2 al 5 del ingreso en la Unidad de Cuidados Intensivos (UCI) (S.Cameron, I. Ball, G. Cepinskas, K. Choong, T. Doherty, C. Ellis, 2015). Ha demostrado ser una práctica segura, factible y beneficiosa en la población adulta, convirtiéndose en una parte importante de la atención del paciente crítico (P. Nydahl, T. Sricharoenchai, S. Chandra, F. Sari, M. Huang, M. Fischill, D. Needham, 2017). No obstante, la práctica de movilización precoz, no ha logrado permear las barreras del equipo multidisciplinario de las Unidades de Cuidados Intensivos Pediátricos (UCIP), según reportan algunos estudios esto se encuentra altamente relacionado con las percepciones del personal sobre la seguridad del paciente durante la movilización (R. Dubb, P. Nydahl,C. Hermes, N. Schwabbauer, A. Toonstra, A. Parker, A. Kaltwasser, D. Needham, 2016). Cabe reconocer que, a diferencia del adulto, existe una complejidad añadida inherente a los grupos etarios y la heterogeneidad de los pacientes que reciben dichas unidades. Esto ha creado una cultura de inmovilización en el pediátrico, la cual está ampliamente relacionada con morbilidades físicas, cognitivas y psiquiátricas en aquellos pacientes que sobreviven a su estancia dentro de dicha unidad, que cabe mencionar, son estos últimos cada vez mayores (Hopkins, Choong, Zebuhr, \& Kudchadkar, 2015). Aunque escasos y pobremente reproducidos, diversos estudios han 
demostrado los beneficios y la factibilidad de implementar un programa de MT en la UCIP (K. Betters, K. Hebbar, D. Farthing, B. Griego, T. Easley, H. Turman, L. Perrino, S. Sparacino, M. DeAlmeida, 2017; B. Wieczorek, C. Burke, A. Al-Harbi, S. Kudchadkar, S. Kudchadkar, 2016). Atañe pues, a los fisioterapeutas y kinesiólogos que se desempeñan dentro de dichas unidades, lograr una conexión entre la evidencia científica y la atención del pediátrico críticamente enfermo, lo cual requiere una comprensión profunda del tema, el grupo diana, su entorno y los obstáculos para el cambio. Comprender estos aspectos le permitirá generar estrategias específicas y contundentes para lograr un cambio sustancial y duradero en la UCIP donde se desempeña. Esto es crucial, pues cada unidad difiere de las demás en relación a la cultura de movilización, los recursos, admisiones y procedimientos de atención (M. Barr, 2010; Grol \& Wensing, 2014; C. Joyce, C. Taipe, B. Sobin, M. Spadaro, B. Gutwirth, L. Elgin, G. Silver, B. Greenwald, C. Traube, 2018).

El objetivo del presente estudio fue evaluar las barreras y los beneficios percibidos por el equipo multidisciplinario de una Unidad de Cuidados Intensivos Pediátricos del Hospital de Especialidades del Niñor y la Mujer de la ciudad mexicana de Querétaro.

\section{Material y Método}

Tipo de estudio: descriptivo transversal

\section{Población de estudio y aplicación del instrumento}

En el periodo de Noviembre-Diciembre del 2018 se aplicó el instumento en el personal del área de Cuidados Críticos Pediátricos del Hospital de Especialidades del Niño y la Mujer "Dr. Felipe Núñez Lara” en la ciudad de Querétaro, México. La UCIP cuenta con un equipo mutlidisciplinario de 30 profesionales de la salud exclusivos para el área. Dicho hospital es un centro de concentración para atención pediátrica en el centro de Mexico. Cuenta con 12 camas de atención critica y 3 de las mencionadas son cúbiculos aislados para pacientes con riesgo de infección. Se procuró encuestar a todo el personal, sin embargo se presentaron casos donde no se quiso participar en la encuesta o no se logró hacer el contacto con el personal. Se logró recabar un total de 28 intrumentos contestados, donde se incluye personal médico, enfermería y de fisioterapia que laboren directamente en la atención del paciente pediátrico críticamente enfermo.

\section{Desarrollo del instrumento}

La encuesta propuesta por Hoyer se basa en las guías de práctica clínica (Cabana et al. 1999). Esta estructura establece como principio que "las bases del personal de las guías de práctica clínica pueden afectar el desenlace de la atención del paciente (E. Hoyer, D. Brotman, K. Chan, D. Needham, 
2016). Esta afectación se centra en tres principales pilares: conocimiento, actitud y comportamiento. (M. Cabana, C. Rand, N. Powe, A. Wu, M. Wilson, P. Abboud, H. Rubin, 1999) Propiamente, el pilar de conocimiento nos habla del adiestramiento y la educación con respecto a la movilización del paciente. Por parte de la actitud se aborda el desacuerdo con la atención, la poca eficiencia y percepciones del proveedor de servicios. El apartado de comportamiento habla de los factores externos y ciertas restricciones que evitan que el proveedor de servicios de salud movilice a los pacientes. (E. Hoyer, et al. 2016) En el caso de Hoyer se recolectaron datos extra como la profesión y los años de experiencia laboral. En la escala aplicada se le implementaron el llenado de datos de sexo, edad y dentro de la profesión el grado de estudios de la misma. Posterior a esto, se realizó la traducción y adaptación a población mexicana la cual fue validada por 5 expertos: 1 médico intensivista, 2 enfermeros especialistas en cuidados críticos y 2 fisioterapeutas con experiencia en cuidados intensivos. En este proceso se obtuvo una unica retrolaimentacion de los expertos en la cual se realizaron los cambios especificados y los comentiarios agregados por los mismos. Ya realizados los cambios, los expertos aprobaron la traduccion y la estructura del instrumento. Se decidió mantener la misma estructura de encuesta que Hoyer y, de igual modo, la escala tiene como método de respuesta 5 posibilidades (1: Desacuerdo completamente, 2: En desacuerdo, 3: Neutral, 4: De acuerdo, 5: De acuerdo completamente). La encuesta tuvo una duración aproximada de 4 y 7 minutos para ser llenada en su totalidad.

\section{Análisis Estadístico}

Se llevó acabo la traducción y validación del instrumento por 5 expertos en cuidados intensivos. Posteriomente se realizó una prueba de fiabilidad por medio del calculo de Alfa de Cronbach general obteniendo un coeficiente de 0.70 y realizando la eliminación de cada uno de los items para verificar la immportancia individual. Se aplicó el instrumento a personal medico y paramedico activo de la UCIP del HENM en todos los turnos de atención. A continuación se vacio la información en una base de datos y se calcularon las frecuencias y porcentajes de las respuestas del instrumento por medio del paquete estadístico IBM SPSS Statistics 21. Se presenta en resultado de manera escrita, en gráficos y tablas.

\section{Resultados}

El estudio se realizó en la Unidad de Cuidados Intensivos Pediátricos del Hospital de Especialidades del Niño y la Mujer (HENM) en la Ciudad mexicana de Querétaro, un hospital de segundo nivel, en el periodo de noviembre a diciembre del año 2018. Se aplicaron 28 cuestionarios a personal 
médico, enfermería y fisioterapia que colaborara en el manejo del paciente crítico de dicha unidad.

El $63 \%$ de los encuestados fueron mujeres y el $37 \%$ hombres con una media de edad general de 32,0 \pm 7,6 años y una experiencia en la UCIP de 2,6 $\pm 3,1$ años. El 75\% del personal encuestada es de enfermaría, los fisioterapeutas son minoría dentro de la UCIP (gráfica 1). El nivel académico del personal fue: $10,7 \%$ técnico, $64,3 \%$ licenciatura y el $25 \%$ posgrado. Se encuestaron a personal de todos los turnos: $28 \%$ matutino, $32 \%$ vespertino, $36 \%$ nocturno y el $4 \%$ turno especial.

La mayoría del personal conocen los beneficios de la movilización y en que momento se debe derivar a los servicios de fisioterapia y terapia ocupacional, además de reconocer la seguridad del procedimiento (tabla 1 y 2).

El $67.86 \%$ del personal encuestado contestó estar de acuerdo y totalmente de acuerdo en que el hospital no cuenta con el equipo y mobilario adecuado para la realización de la movlización, siendo la principal barrera para su realización (Tabla 3).

El personal de enfermería moviliza al paciente al menos una vez al día como parte de los cuidados generales dentro de la UCIP. La movilización en esta unidad parece ser bien aceptada por el personal médico y paramédico, además se reconoce la importancia que dicha intervención sea realizada por un fisioterapeuta o terapeuta ocupacional principalmente.

Por otro lado se valoró fiabilidad del instrumento por medio de Alpha de Cronbach y se realizó una evaluación elimando cada uno de los items para verificar su importancia individual en el instrumento. Adicionalmente se realizó una correlación bivariada de Pearson de la sumataria con cada uno de los items para verificar factores de correlación, teniendo una significancia estadística aquellos con una $\mathrm{p}=<0,05$ (tabla 4 ).

Gráfica 1. Personal encuentado en la UCIP según profesión

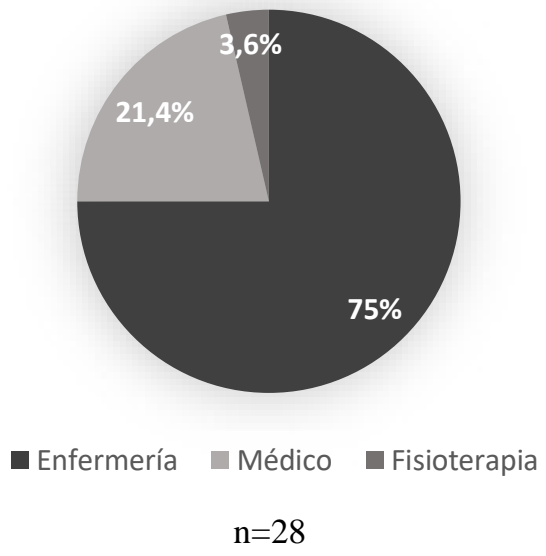


Tabla 1. Barreras de la movilización temprana subescala: conocimiento Distribución de las respuestas en \%

\begin{tabular}{|c|c|c|c|c|c|c|c|c|}
\hline Subescala & $\begin{array}{c}\text { No. de } \\
\text { item }\end{array}$ & Item & 1 & 2 & 3 & 4 & 5 & $\begin{array}{c}\text { Media } \\
( \pm)\end{array}$ \\
\hline \multirow[t]{4}{*}{ Conocimiento } & 2 & $\begin{array}{l}\text { He recibido } \\
\text { entrenamiento de } \\
\text { como movilizar a } \\
\text { mis pacientes }\end{array}$ & 21,43 & 14,29 & 7,14 & 39,29 & 17,86 & $\begin{array}{c}3,1 \\
(1,4)\end{array}$ \\
\hline & 5 & $\begin{array}{lr}\text { Entiendo } & \text { que } \\
\text { pacientes } & \text { son } \\
\text { apropiados } & \text { para } \\
\text { derivar a Fisioterapia }\end{array}$ & 0 & 7,14 & 17,86 & 42,86 & 32,14 & $\begin{array}{c}4,0 \\
(0,9)\end{array}$ \\
\hline & 6 & $\begin{array}{lr}\text { Entiendo } & \text { que } \\
\text { pacientes } & \text { son } \\
\text { apropiados } & \text { para } \\
\text { referir a } & \text { Terapia } \\
\text { Ocupacional } & \end{array}$ & 0 & 25 & 14,29 & 28,57 & 32,14 & $\begin{array}{c}3,6 \\
(1,1)\end{array}$ \\
\hline & 25 & $\begin{array}{l}\text { Al menos que exista } \\
\text { una contraindicación } \\
\text { yo educo a mis } \\
\text { pacientes para que se } \\
\text { ejerciten o aumenten } \\
\text { sus actividades } \\
\text { físicas en mi unidad } \\
\text { hospitalaria }\end{array}$ & 7,14 & 7,14 & 10,71 & 39,29 & 35,71 & $\begin{array}{c}3,8 \\
(1,1)\end{array}$ \\
\hline
\end{tabular}

1= Totalmente en desacuerdo, $2=$ Desacuerdo, $3=$ Neutral, $4=$ De acuerdo, $5=$ Totalmente de acuerdo $\mathrm{n}=28$ 
Tabla 2. Barreras de la movilización temprana subescala: actitud Distribución de las respuestas en $\%$

\begin{tabular}{|c|c|c|c|c|c|c|c|c|}
\hline Subescala & $\begin{array}{l}\text { No. de } \\
\text { item }\end{array}$ & Item & 1 & 2 & 3 & 4 & 5 & $\begin{array}{l}\text { Medi } \\
\mathrm{a}( \pm)\end{array}$ \\
\hline \multirow[t]{10}{*}{ Actitud } & 1 & $\begin{array}{l}\text { Mis pacientes están } \\
\text { demasiado enfermos } \\
\text { para ser movilizados }\end{array}$ & 14,29 & 28,57 & 28,57 & 28,57 & 0 & $\begin{array}{c}2,1 \\
(1,0)\end{array}$ \\
\hline & 3 & $\begin{array}{l}\text { Incrementar la } \\
\text { movilización de mis } \\
\text { pacientes será dañino } \\
\text { para ellos }\end{array}$ & 32,14 & 28,57 & 28,57 & 7,14 & 3,57 & $\begin{array}{c}2,2 \\
(1,1)\end{array}$ \\
\hline & 4 & 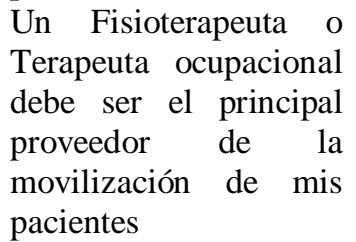 & 7,14 & 17,86 & 28,57 & 21,43 & 25,0 & $\begin{array}{c}3,3 \\
(1,2)\end{array}$ \\
\hline & 12 & $\begin{array}{l}\text { Aumentar la } \\
\text { movilización de los } \\
\text { pacientes generara más } \\
\text { carga de trabajo a } \\
\text { enfermería }\end{array}$ & 17,86 & 46,43 & 17,86 & 3,57 & 14,29 & $\begin{array}{c}2,5 \\
(1,2)\end{array}$ \\
\hline & 13 & $\begin{array}{lr}\text { Aumentar } & \text { la } \\
\text { movilización de los } \\
\text { pacientes generara más } \\
\text { carga de trabajo los } \\
\text { Fisioterapeutas } \\
\text { Terapeutas } \\
\text { ocupacionales }\end{array}$ & 25 & 42,86 & 14,29 & 17,86 & 0 & $\begin{array}{c}2,2 \\
(1,0)\end{array}$ \\
\hline & 18 & $\begin{array}{l}\text { Creo que a los pacientes } \\
\text { que están siendo } \\
\text { movilizados al menos } 2 \\
\text { veces al día tienen un } \\
\text { mejor } \\
\text { hospitalario }\end{array}$ & 0 & 0 & 10,71 & 35,71 & 53,57 & $\begin{array}{c}4,4 \\
(0,6)\end{array}$ \\
\hline & 19 & $\begin{array}{l}\text { Desconozco cuando es } \\
\text { seguro movilizar a los } \\
\text { pacientes }\end{array}$ & 35,71 & 42,86 & 14,29 & 3,57 & 3,57 & $\begin{array}{c}1,9 \\
(0,9)\end{array}$ \\
\hline & 21 & $\begin{array}{l}\text { No siento confianza con } \\
\text { mi habilidad de }\end{array}$ & 25 & 46,43 & 17,86 & 10,71 & 0 & $\begin{array}{c}2,1 \\
(0,9)\end{array}$ \\
\hline & & $\begin{array}{l}\text { movilizar } \\
\text { pacientes }\end{array}$ & & & & & & \\
\hline & 26 & $\begin{array}{l}\text { Mis pacientes tienen } \\
\text { tiempo en su día de ser } \\
\text { movilizados al menos } \\
\text { tres veces al día }\end{array}$ & 0 & 7,14 & 25 & 21,43 & 46,43 & $\begin{array}{c}4,0 \\
(1,0)\end{array}$ \\
\hline
\end{tabular}

1= Totalmente en desacuerdo, $2=$ Desacuerdo, $3=$ Neutral, $4=$ De acuerdo, $5=$ Totalmente de acuerdo $\mathrm{n}=28$ 
Tabla 3. Barreras de la movilización temprana subescala: comportamiento Distribución de las respuestas en \%

\begin{tabular}{|c|c|c|c|c|c|c|c|c|}
\hline Subescala & $\begin{array}{l}\text { No. } \\
\text { item }\end{array}$ & de Item & 1 & 2 & 3 & 4 & 5 & $\begin{array}{l}\text { Media } \\
( \pm)\end{array}$ \\
\hline \multirow[t]{10}{*}{$\begin{array}{l}\text { Comportamie } \\
\text { nto }\end{array}$} & 7 & $\begin{array}{l}\text { No tenemos el equipo } \\
\text { apropiado o mobiliario para } \\
\text { movilizar a pacientes }\end{array}$ & $\begin{array}{c}7,1 \\
4\end{array}$ & 14,29 & 10,71 & 50 & 17,86 & $\begin{array}{c}3,5 \\
(1,1)\end{array}$ \\
\hline & 8 & $\begin{array}{l}\text { La funcionalidad física de } \\
\text { mis pacientes es discutida } \\
\text { con regularidad entre los } \\
\text { proveedores de salud del } \\
\text { paciente }\end{array}$ & $\begin{array}{c}3,5 \\
7\end{array}$ & 32,14 & 35,71 & 25 & 3,57 & $\begin{array}{l}2,9 \\
(0,9)\end{array}$ \\
\hline & 9 & $\begin{array}{l}\text { El personal en relación } \\
\text { enfermero-paciente es } \\
\text { adecuado para movilizar } \\
\text { pacientes en mi unidad }\end{array}$ & 0 & 21,43 & 17,86 & 53,57 & 7,14 & $\begin{array}{c}3,4 \\
(0,9)\end{array}$ \\
\hline & 10 & $\begin{array}{l}\text { Mis pacientes a menudo } \\
\text { tienen contraindicaciones } \\
\text { para ser movilizados }\end{array}$ & $\begin{array}{l}10 \\
71\end{array}$ & 28,57 & 35,71 & 21,43 & 3,57 & $\begin{array}{c}2,7 \\
(1,0)\end{array}$ \\
\hline & 11 & $\begin{array}{l}\text { Al menos que existan } \\
\text { contraindicaciones, mis } \\
\text { pacientes son movilizados } \\
\text { al menos una vez por } \\
\text { enfermería }\end{array}$ & $\begin{array}{c}3,5 \\
7\end{array}$ & 3,57 & 3,57 & 46,43 & 42,86 & $\begin{array}{c}4,2 \\
(0,9)\end{array}$ \\
\hline & 14 & $\begin{array}{lr}\text { El liderazgo de mi } \\
\text { departamento } \\
\text { fuertemente } \\
\text { movilización de pacientes }\end{array}$ & 0 & 7,14 & 28,57 & 28,57 & 35,71 & $\begin{array}{c}3,9 \\
(0,9)\end{array}$ \\
\hline & 15 & $\begin{array}{l}\text { Incrementar la } \\
\text { movilización aumenta el } \\
\text { riesgo de lesiones en mis } \\
\text { pacientes }\end{array}$ & $\begin{array}{l}35 \\
71\end{array}$ & 35,71 & 7,14 & 10,71 & 10,71 & $\begin{array}{c}2,2 \\
(1,3)\end{array}$ \\
\hline & 16 & $\begin{array}{l}\text { Pacientes que pueden ser } \\
\text { movilizados usualmente } \\
\text { tienen órdenes médicas } \\
\text { para la movilización }\end{array}$ & $\begin{array}{c}3,5 \\
7\end{array}$ & 21,43 & 28,57 & 39,29 & 7,14 & $\begin{array}{c}3,2 \\
(1,0)\end{array}$ \\
\hline & 17 & $\begin{array}{l}\text { Mis pacientes se resisten a } \\
\text { ser movilizados }\end{array}$ & 25 & 60,71 & 10,71 & 3,57 & 0 & $\begin{array}{l}1,9 \\
(0,7)\end{array}$ \\
\hline & 20 & $\begin{array}{l}\text { Los familiares de mis } \\
\text { pacientes normalmente } \\
\text { están interesados en ayudar } \\
\text { a movilizarlos }\end{array}$ & $\begin{array}{l}10 \\
71\end{array}$ & 14,29 & 17,86 & 28,57 & 28,57 & $\begin{array}{c}3,5 \\
(1,3)\end{array}$ \\
\hline
\end{tabular}

1= Totalmente en desacuerdo, 2= Desacuerdo, 3= Neutral, 4= De acuerdo, 5= Totalmente de acuerdo $\mathrm{n}=28$ 
Tabla 4. Nivel de fiabilidad por item y correlación con sumatoria total.

\begin{tabular}{|c|c|c|c|}
\hline No. & Item & $\begin{array}{l}\text { Alfa de Cronbach si se } \\
\text { elimina el elemento }\end{array}$ & $\begin{array}{l}\text { Correlación de } \\
\quad \text { Pearson }\end{array}$ \\
\hline 1 & $\begin{array}{l}\text { Mis pacientes están demasiado enfermos para } \\
\text { ser ejercitados }\end{array}$ & 0,69 & 0,329 \\
\hline 2 & $\begin{array}{l}\text { He recibido entrenamiento de como ejercitar a } \\
\text { mis pacientes }\end{array}$ & 0,69 & $0,436^{*}$ \\
\hline 3 & $\begin{array}{l}\text { Incrementar el ejercicio para mis pacientes será } \\
\text { dañino para ellos }\end{array}$ & 0,69 & 0,344 \\
\hline 4 & $\begin{array}{l}\text { Un fisioterapeuta o terapeuta ocupacional debe } \\
\text { ser el principal proveedor del ejercicio de mis } \\
\text { pacientes }\end{array}$ & 0,71 & 0,199 \\
\hline 5 & $\begin{array}{l}\text { Entiendo que pacientes son apropiados para } \\
\text { derivar a fisioterapia }\end{array}$ & 0,69 & 0,353 \\
\hline 6 & $\begin{array}{l}\text { Entiendo que pacientes son apropiados para } \\
\text { referir a terapia ocupacional }\end{array}$ & 0,69 & 0,351 \\
\hline 7 & $\begin{array}{l}\text { No tenemos el equipo apropiado o mobiliario } \\
\text { para ejercitar al paciente }\end{array}$ & 0,67 & $0,613^{* *}$ \\
\hline 8 & $\begin{array}{l}\text { La funcionalidad de mis pacientes es discutida } \\
\text { con regularidad entre los proveedores de salud } \\
\text { del paciente }\end{array}$ & 0,71 & 0,035 \\
\hline 9 & $\begin{array}{l}\text { El personal en relación enfermero-paciente es } \\
\text { adecuado para ejercitar a los pacientes en mi } \\
\text { unidad }\end{array}$ & 0,70 & 0,167 \\
\hline 10 & $\begin{array}{l}\text { Mis pacientes a menudo } \\
\text { contraindicaciones para ser ejercitados }\end{array}$ & 0,72 & 0,041 \\
\hline 11 & $\begin{array}{l}\text { Al menos que tengan contrainidicaciones, mis } \\
\text { pacientes son ejercitados al menos } 1 \text { vez al día } \\
\text { por enfermería }\end{array}$ & 0,69 & 0,338 \\
\hline 12 & $\begin{array}{l}\text { Aumentar el ejercicio de los pacientes generará } \\
\text { más carga de trabajo a enfermería }\end{array}$ & 0,69 & 0,371 \\
\hline 13 & $\begin{array}{l}\text { Aumentar el ejercicio de los pacientes generara } \\
\text { más carga de trabajo a los fisioterapeutas y/o } \\
\text { terapeutas ocupacionales }\end{array}$ & 0,71 & 0,160 \\
\hline 14 & $\begin{array}{l}\text { El liderazgo de mi departamento apoya } \\
\text { fuertemente el ejercicio en los pacientes }\end{array}$ & 0,67 & $0,569^{* *}$ \\
\hline 15 & $\begin{array}{l}\text { Incrementar la frecuencia del ejercicio aumenta } \\
\text { el riesgo de lesiones en mis pacientes }\end{array}$ & 0,70 & 0,277 \\
\hline 16 & $\begin{array}{l}\text { Pacientes que pueden ser ejercitados usualmente } \\
\text { tienen órdenes médicas apropiadas para } \\
\text { ejercitarse }\end{array}$ & 0,70 & 0,237 \\
\hline 17 & Mis pacientes se resisten a ser ejercitados & 0,70 & 0,114 \\
\hline 18 & $\begin{array}{l}\text { Creo que los pacientes que estan siendo } \\
\text { ejercitados al menos } 3 \text { veces al día tiene un } \\
\text { mejor egreso hospitalario }\end{array}$ & 0,69 & $0,380^{*}$ \\
\hline 19 & $\begin{array}{l}\text { Desconozco cuando es seguro ejercitar a los } \\
\text { pacientes }\end{array}$ & 0,70 & 0,209 \\
\hline 20 & $\begin{array}{l}\text { Los familiares de mis pacientes normalmente } \\
\text { están interesados en ayudar a ejercitarlos }\end{array}$ & 0,68 & $0,454^{*}$ \\
\hline
\end{tabular}


$21 \quad$ No siento confianza con mi habilidad de ejercitar a mis pacientes

22 Yo documento el estado de la funcionalidad

física de mi paciente durante mi turno o día de trabajo

23 Yo no tengo tiempo de ejercitar a mis pacientes en turno o día de trabajo

24 Al menos que exista contraindicación, yo ejercito a mis pacientes al menos 1 vez en mi turno o día de trabajo

$25 \mathrm{Al}$ menos que exista contraindicación, yo educo a mis pacientes para que se ejerciten o aumenten

$$
0,68 \quad 0,505^{* *}
$$
su actividad física en mi unidad hospitalaria

26 Mis pacientes tienen tiempo en su día de ser ejercitados al menos 3 veces al día

\section{Discusion}

En las UCIP las barreras actitudinales involucran aspectos personales e interpersonales difíciles de resolver. Sin embargo, según los datos obtenidos, en el HENM se percibe un campo fértil para la germinación de un programa de MT ya que los resultados obtenidos fueron más que positivos. Donde el grueso de los encuestados considera que la MT es benéfica y que sus pacientes normalmente no se encuentran demasiado enfermos como para no ser movilizados y tanto los pacientes como los familiares se muestran cooperativos. De hecho, reconocen que los pediátricos que son sometidos $2 \mathrm{o}$ más veces al día a actividad física presentan un mejor egreso hospitalario. Cabe mencionar que no consideran que aumentar la movilización de pacientes generará más carga de trabajo para el personal de enfermería ya que, sorpresivamente, admiten que debe ser un Fisioterapeuta o Terapeuta ocupacional el principal proveedor de esta práctica.

El ítem negativo de mayor relevancia fue el número siete, donde se constata que el $67.86 \%$ de los encuestados consideran que el equipo y mobiliario existente no es el adecuado para implementar un programa de MT. Existen antecedentes donde se señala a la falta de material como una barrera para la MT y deambulación de pacientes por parte del personal de enfermerìa (Brown, Williams, Woodby, Davis, \& Allman, 2007). Y, aunque existe una amplia gama de equipos de rehabilitación adecuados para el paciente de la UCIP, es ampliamente aceptado que al fisioterapeuta le es más benéfico para su intervención una buena coordinación con el equipo multidiciplinario y suficiente recurso humano, en lugar de un equipo sofisticado de movilización y deambulación del paciente.

Se ha documentado que incluso en situaciones similares de poco personal disponible, la adquisición de equipo y mobiliario orientado a ayudar 
a la progresión de movilidad en pacientes de la UCI, no es tan efectiva para la MT como la presencia de un protocolo de MT para aumento de la frecuencia de la actividad fuera de la cama. Demostrando que el personal de enfermería puede iniciar con éxito un programa de MT aún con recursos limitados, apoyándose de un protocolo que lo guíe en la toma de decisiones. (Peereboom, 2015)

Sin embargo, parece ser que el siguiente paso a tomar en el HENM es la realización de un modelo financiero que permita gestionar con las autoridades del hospital la compra del equipo necesario y probablemente el reclutamiento de más personal de Fisioterapia y/o terapia ocupacional.

En el 2013, se publicó un estudio de costo-beneficio de hospitales en Estados Unidos donde se implementó un programa de MT en adultos, El modelo consideró las siguientes variables: 1) la reducción en la estancia hospitalaria lograda por el programa, 2) el ahorro por día debido a la disminución de la estancia hospitalaria, 3) los costos de implementación del programa, y 4) el número anual de admisiones en la UCI. De este, concluyeron que la inversión en un programa de movilización temprana de la UCI puede generar ahorros financieros anuales netos. (R. Lord, C. Mayhew, R. Korupolu, E. Mantheiy, M. Friedman, J. Palmer, D. Needham, 2013)

Este estudio tuvo varias limitaciones. El estudio se realizó en una sola institución con una muestra por conveniencia. Hay que tomar en cuenta que se trata de un hospital de especialidades, por lo que puede que no se apliquen los resultados aquí demostrados a entornos de hospitales comunitarios o entre personal con diferentes atributos. Sin embargo, este estudio proporciona datos únicos sobre las percepciones del personal multidiciplinario que ahí labora.

El estudio arrojó resultados muy positivos, ya que sólo el $35.72 \%$ del personal encuestado notificó no contar con un entrenamiento apropiado para movilizar a los pacientes de la UCIP. Sin embargo, este dato no representa una barrera potencial para la MT según los autores, ya que la mayoría del personal encuestado se manifestó capacitado para identificar qué pacientes deben de ser referidos al área de Fisioterapia y/o Terapia Ocupacional. Aunado a esto, más del $85.71 \%$ refiere promover actualmente la actividad física en sus pacientes durante la estancia hospitalaria esto nos sugiere que, de existir, habría buena adhesión del pediátrico y los familiares a un programa de MT en la UCIP del HENM.

Según lo que reporta el personal encuestado, invertir en mobiliario y material para la realización de los programas de MT propiciará la reducción en la inmovilidad de los pacientes y se obtendrán todos los beneficios ya comentados con respecto a la MT. Además, al lograr romper con estas barreras, aumentaría de forma amplia el campo de la investigación, abriendo las puertas para continuar indagando en el campo intensivista y la fisioterapia en conjunto. 


\section{Conclusion}

Preocuparse por un egreso funcional del paciente es hablar de atención de alta calidad, en especial con poblaciones pediátricas. El poner de manifiesto las barreras reportadas por el personal, nos da oportunidad de atacarlas desde un enfoque más específico y dirigido por metas. En este caso, el planteamiento y la gestión, tanto de mobiliario como de equipo para realización de las prácticas de MT, tendrá que ser la estrategia a seguir. Por lo tanto, la creación de un programa de movilización temprana parece un plan sumamente viable y posible de desarrollar en la UCIP del HENM. De esta manera, la práctica fisioterapéutica podrá ser respaldada de forma adecuada como una intervención cotidiana y necesaria.

Finalmente, instamos a todos los profesionales que se desempeñan en la UCIP a reconocer las barreras que presenta su unidad respecto a la MT y así, poder hacer estudios multicéntricos a nivel nacional que permitan identificar el estado actual de dicha práctica en México. Y en un futuro cercano, poder iniciar con ensayos clínicos que describan el impacto de dicha practica en población mexicana.

\section{References:}

1. Barr, M. (2010). The iron lung - a polio patient's story. Jrsm, 103(6), 256-259. https://doi.org/10.1258/jrsm.2010.100003

2. Betters, K. A., Hebbar, K. B., Farthing, D., Griego, B., Easley, T., Turman, H., ... deAlmeida, M. L. (2017). Development and implementation of an early mobility program for mechanically ventilated pediatric patients. Journal of Critical Care, 41, 303-308. https://doi.org/10.1016/j.jcrc.2017.08.004

3. Brown, C. J., Williams, B. R., Woodby, L. L., Davis, L. L., \& Allman, R. M. (2007). Barriers to Mobility During Hospitalization from the Perspectives of Older Patients and Their Nurses and Physicians, 305313. https://doi.org/10.1002/jhm.209

4. Cabana MD, Rand CS, Powe NR, et al. (1999). Why Don ' $t$ Physicians Follow A Framework for Improvement, 718.

5. Cameron, S., Ball, I., Cepinskas, G., Choong, K., Doherty, T. J., Ellis, C. G., ... Fraser, D. D. (2015). Early mobilization in the critical care unit: A review of adult and pediatric literature. Journal of Critical Care, 30(4), 664-672. https://doi.org/10.1016/j.jcrc.2015.03.032

6. Dubb, R., Nydahl, P., Hermes, C., Schwabbauer, N., Toonstra, A., Parker, A. M., ... Needham, D. M. (2016). Barriers and Strategies for Early Mobilization of Patients in Intensive Care Units, 13(5). https://doi.org/10.1513/Annals ATS.201509-586CME

7. Erik H. Hoyer, et al. (2016). Barriers to Early Mobility of Hospitalized General Medicine Patients, 70(12), 773-779. 
https://doi.org/10.1097/OGX.0000000000000256.Prenatal

8. Grol, R., \& Wensing, M. (2014). What drives change ? Barriers to and incentives for achieving evidence-based practice, (May).

9. Hopkins, R., Choong, K., Zebuhr, C., \& Kudchadkar, S. (2015). Transforming PICU Culture to Facilitate Early Rehabilitation. Journal of Pediatric Intensive Care, 4(4), 204-211. https://doi.org/10.1055/s0035-1563547

10. Joyce, C. L., Taipe, C., Sobin, B., Spadaro, M., Gutwirth, B., Elgin, L., ... Traube, C. (2018). Provider Beliefs Regarding Early Mobilization in the Pediatric Intensive Care Unit. Journal of Pediatric Nursing, 38, 15-19. https://doi.org/10.1016/j.pedn.2017.10.003

11. Lord, R. K., Mayhew, C. R., Korupolu, R., Mantheiy, E. C., Friedman, M. A., Palmer, J. B., \& Needham, D. M. (2013). ICU Early Physical Rehabilitation Programs. Critical Care Medicine, 41(3), 717-724. https://doi.org/10.1097/CCM.0b013e3182711de2

12. Nydahl, P., Sricharoenchai, T., Chandra, S., Sari, F., Ma, M. P. H., Huang, M., ... Needham, D. M. (2017). Safety of Patient Mobilization and Rehabilitation in the ICU: Systematic Review with MetaAnalysis, 6, 1-67.

13. Peereboom, K. (2015). Staff-Perceived Barriers and Facilitators, $30(2)$.

14. Wieczorek, B., Ascenzi, J., Kim, Y., Lenker, H., Potter, C., Shata, N. J., ... Kudchadkar, S. R. (2016). PICU Up! Pediatric Critical Care Medicine, $17(12)$, e559-e566. https://doi.org/10.1097/PCC.0000000000000983 